\title{
Pre-Surgical Endovascular Proximal Feeder Artery Devascularization Technique for the Treatment of Cranial Arteriovenous Malformations
}

This article was published in the following Dove Press journal:

Vascular Health and Risk Management

\author{
Khaled Alawneh (1D' \\ Bashar Abuzayed ${ }^{2}$ \\ Majdi Al Qawasmeh ${ }^{3}$ \\ Liqaa Raffee $\mathbb{D I}^{4}$ \\ Abdelwahab Aleshawi ${ }^{5}$ \\ 'Department of Radiology, Faculty of \\ Medicine, Jordan University of Science \\ and Technology, Irbid 22II0, Jordan; \\ ${ }^{2}$ Department of Neurosurgery, The \\ Specialty Hospital, Amman, Jordan; \\ ${ }^{3}$ Department of Neuroscience, Division \\ of Neurology, Faculty of Medicine, Jordan \\ University of Science and Technology, \\ Irbid 22II0, Jordan; ${ }^{4}$ Department of \\ Accident and Emergency, Faculty of \\ Medicine, Jordan University of Science \\ and Technology, Irbid 22I I0, Jordan; \\ ${ }^{5}$ King Abdullah University Hospital, \\ Jordan University of Science and \\ Technology, Irbid, Jordan
}

Objective: Treatment of brain arteriovenous malformations (AVMs) aims to abolish any risk for intracranial hemorrhage with the preservation of the patient's functional status. We present the technique of pre-surgical endovascular devascularization through proximal feeder artery occlusion for the treatment of cranial AVMs rather than nidus occlusion. Also, we highlight the advantages and the possible clinical indications.

Patients and Methods: Two patients with brain AVM and one patient with scalp AVM were treated by pre-surgical endovascular devascularization followed by surgical resection. Endovascular devascularization was performed by occlusion of the AVM feeders only with Liquid Embolic System Agent (Onyx ${ }^{\circledR}$ ) 18 without entering and filling the nidus. During surgery, feeding arteries colored with the black color of the Liquid Embolic System Agent were clearly identified and cut. Dissection of the AVM was performed, and resection of the nidus was achieved.

Results: Total resection of the AVM was achieved in all cases confirmed with follow-up angiographies, with no neurologic or systemic complications. Also, no major bleeding was detected. In addition, the surgical clips were avoided during surgery. Brain AVMs were safely resected in piecemeal fashion.

Conclusion: Pre-surgical endovascular proximal feeder artery devascularization technique shows to be a safe, simple and effective technique for the management of cranial arteriovenous malformations. This technique simplifies both the endovascular and surgical approaches to complicated cranial AVM cases.

Keywords: angiography, arteriovenous malformation, devascularization, endovascular, surgical resection

\section{Introduction}

Cerebral arteriovenous malformations (AVMs) are uncommon intracranial lesions usually presenting as brain hemorrhage with an annual risk of bleeding of $2-4 \%{ }^{1}$ The main aim of the treatment of AVMs is to reduce or eliminate the risk of intracranial hemorrhage and to preserve or restore the patient's functional status. ${ }^{1,2}$ Their management presents a technical challenge. AVMs are still challenging vascular lesions because they are a heterogeneous pathological entity, varying in their shape, location, size, and hemodynamics, with an unpredictable, despite developments in endovascular, surgical, and microsurgical management modalities. ${ }^{2}$ The treatment of choice is microsurgical resection because it can achieve a selective AVM resection because it excludes the AVM immediately and definitively. ${ }^{3}$ For patients with a high risk of
Correspondence: Khaled Alawneh Department of Radiology, Faculty of Medicine, Jordan University of Science and Technology, Irbid, Jordan $\mathrm{Tel}+962795314555$

Email kzalawneh0@just.edu.jo 
morbidity, endovascular approach, radiosurgery, or multidisciplinary combined approaches are options to be considered. ${ }^{1,2}$

In this article, we present our technique of pre-surgical proximal artery endovascular devascularization for the treatment of cranial AVMs with discussion of the indications and the favorable features of this technique with practical application in demonstrative cases.

\section{Patients and Methods}

Two patients with bleeded brain AVM and one patient with scalp AVM were treated by pre-surgical endovascular devascularization followed by surgical resection. The main principle of pre-surgical endovascular AVM embolization is to occlude only the feeding arteries to decreases the intranidal blood flow and volume without the need to enter and embolize the nidus. In this way, we facilitate the intra-surgical planning and resection of the nidus by previous occlusion and marking (coloring) of the feeding arteries for the surgeon before resection, instead of the intra-surgical investigation, testing, and clipping of the patent feeders (Figure 1A-C).

After performing diagnostic 6-vessel cerebral angiography, a detailed study of the vascular architecture is done and main arterial feeders are identified to be targeted for endovascular occlusion. Very small feeders difficult to access and thought to be with minimal impact upon nidus feeding can be excluded. Planning of the occlusion also includes the site of the occlusion of the marked arteries in a way to be as proximal as possible to the nidus without being an arterial branch supplying normal brain tissue distal to the occlusion site. The patient then is operated under general anesthesia by endovascular technique to inject Liquid Embolic System Agent (LESA) (Onyx ${ }^{\circledR} 18$, Micro-Therapeutics, Inc., Irvine, CA) slowly to occlude the marked feeding arteries and visualize significant reduction of blood flow inside the nidus. Onyx ${ }^{\circledR}$ or LESA is a non-adhesive liquid embolic agent comprising ethylene vinyl alcohol copolymer dissolved in dimethyl sulfoxide and suspended micronized tantalum powder to provide contrast for visualization under fluoroscopy. Options about the timing to carry on surgical resection are variable. The patient can be operated surgically directly after the endovascular de-vascularization under the same anesthesia or surgery can be delayed but not more than few days.

Under general anesthesia and proper position of the patient according to the location of the AVM craniotomy is performed. No large craniotomies extending far beyond the limits of the AVM are needed for tracking the feeder arteries. Upon the previous knowledge of the angiographic anatomy
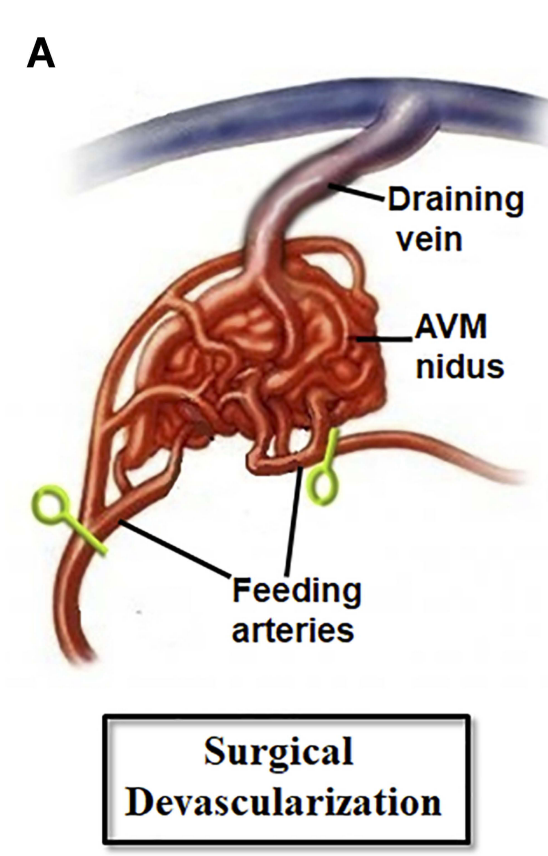

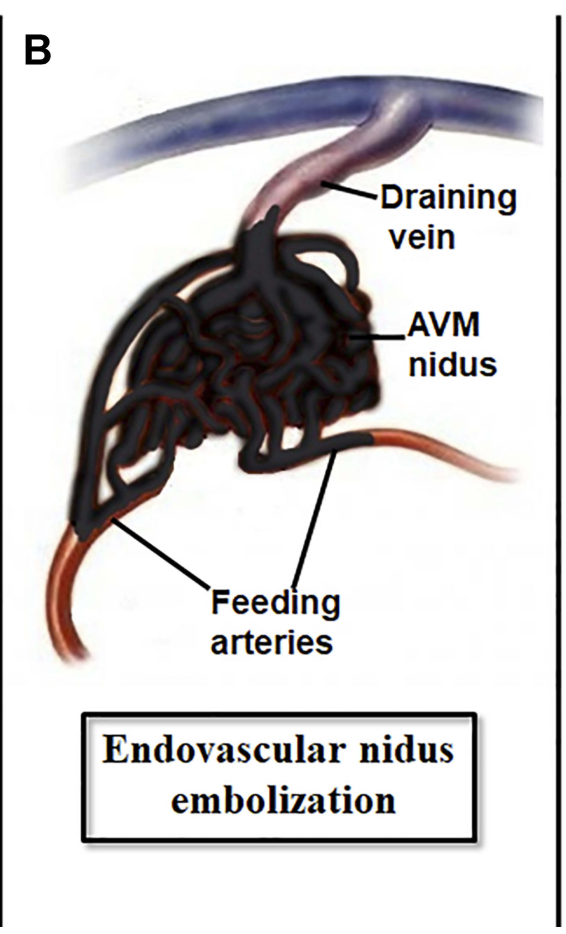

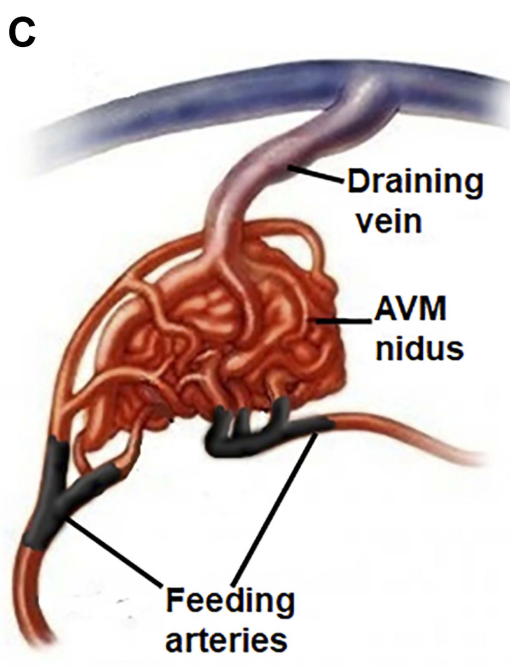

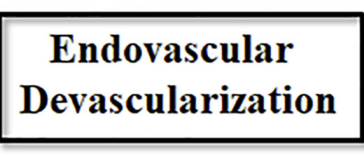

Figure I Schematic drawing demonstrating various approaches to AVM. (A) Intra-operative clip or coagulation devascularization during surgical resection. (B) Endovascular nidus embolization. (C) Endovascular feeders occlusion (devascularization) prior surgical resection. 
of the AVM and the site of the occluded arteries, direct dissection toward these arteries can be performed safely. First, the risk of bleeding is significantly decreased and the AVM becomes shrinked. Also, and very importantly, no need for detailed dissection of the feeding arteries and test them with occlusion test or intraoperative indocyanine green angiography (ICG) because these arteries are already marked with the dark color of the LESA. These dark colored arteries are already occluded and known to be the main feeders; therefore, safe cutting and separation of these arteries can be done. Also, during the circumferential dissection of the nidus when these feeders are not exposed yet, safe coagulation of the nidus and even of the main venous drainage can be done as the AVM is already de-vascularized similar to any AVM after clipping the feeding arteries. Moreover, in cases of large, bulky and irregular shaped AVM's, the resection in piecemeal fashion becomes feasible with this technique and the overall operation becomes similar to the resection of low vascularized tumors. Thus, offering more safe and short-time surgery for the patient.

\section{Case I}

A 17-year-old male patient was first referred to emergency service 8 years ago with a complaint of sudden onset loss of consciousness at the age of 9 years. Brain computed tomography (CT) scan and magnetic resonance imaging (MRI) revealed intracerebral and intraventricular hemorrhage with acute hydrocephalus. Six-vessel cerebral angiography was performed and demonstrated large splenium AVM supplied from distal branches of A5 segment of left anterior cerebral artery (ACA) and the lateral posterior choroidal artery branch of the left posterior cerebral artery (PCA). The venous drainage was seen directly into the vein of Galen (Spetzler-Martin Grade IV) (Figure 2A-D). At that time, external ventricular drain was placed followed by ventriculoperitoneal shunt. No surgical intervention for the AVM was performed at that time. For 8 years, the patient suffered from recurrent bleeding with an average of 1 bleed a year and mean hospital stay of 3 months/year. At the end, the patient was referred to our outpatient clinic and we decided to
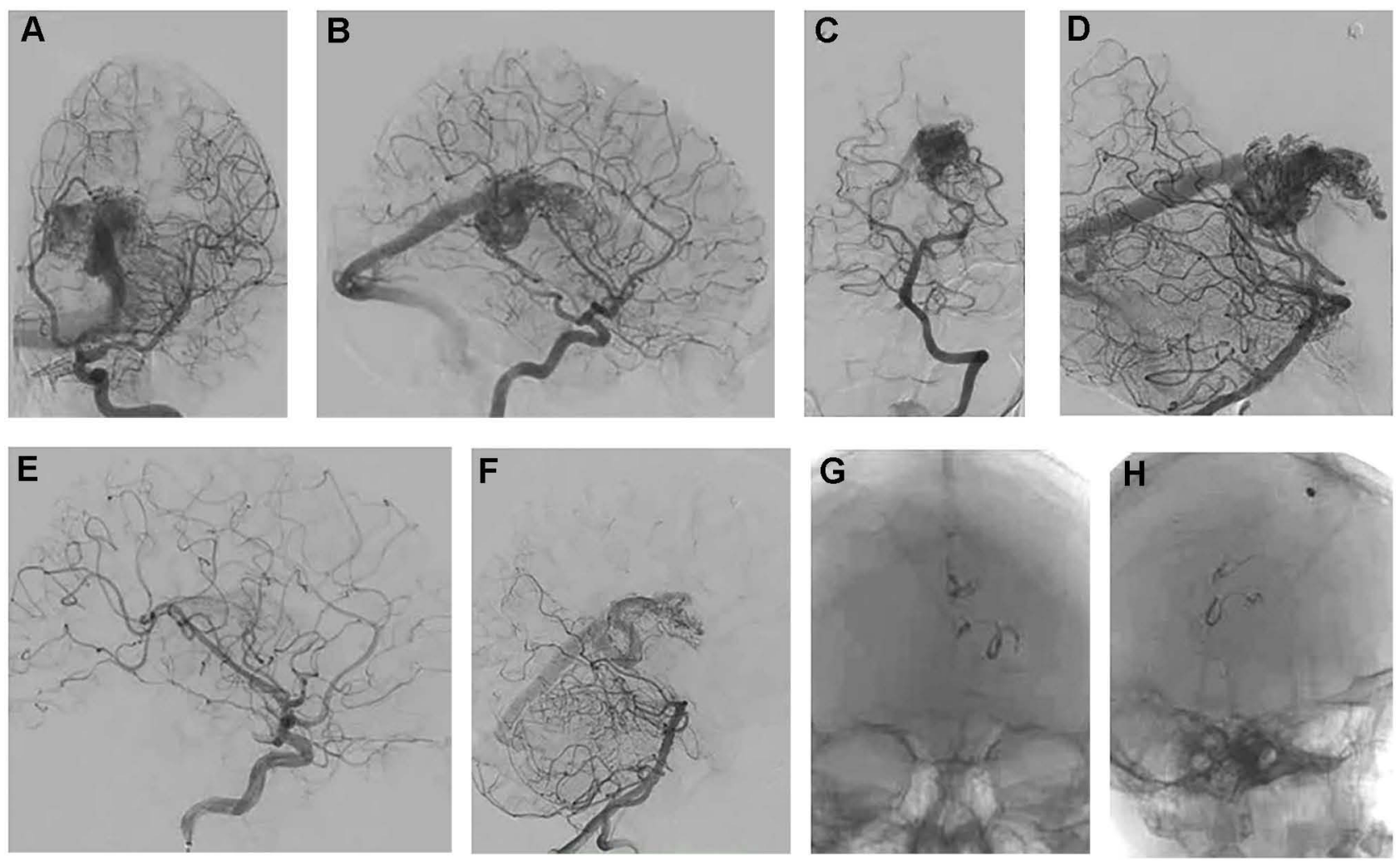

Figure 2 (A) PA left ICA, (B) lateral left ICA, (C) PA left basilar artery, and (D) lateral left basilar artery angiography showing the morphology of the splenium AVM supplied by the $\mathrm{A} 5$ segment of the left ACA and the lateral posterior choroidal artery branch of the left PCA and draining into the vein of Galen. (E) Lateral left ICA and (F) lateral left basilar artery angiography after occlusion of the feeding artery showing the significant regress of the AVM. (G) PA and (H) lateral skull X-rays showing the location of the Onyx ${ }^{\circledR}$ and devascularization sites. 
operate the patient with pre-surgical endovascular devascularization technique followed by surgical resection.

Under general anesthesia, and through endovascular approach by right femoral artery access, AVM feeder from the left A5 segment and left lateral posterior choroidal artery were sealed with LESA. Post-embolization, brain angiography revealed regression of the nidus (Figure 2E-H). Three days after, the patient was operated with neuronavigationguided posterior left interhemispheric approach. During the surgery, the feeder artery was identified with its dark color marked by LESA and cut. Circumferential dissection of the AVM was performed with minimal bleeding and the AVM was resected totally in piecemeal fashion with no vascular clips and with estimated blood loss of about $250 \mathrm{~mL}$ (Figure 3A-C). Postoperatively, the patient had uneventful recovery with no post-operative complications. After oneyear postoperatively, cerebral angiography was done and showed total resection of the AVM (Figure 3D-H).

\section{Case 2}

A 5-year-old boy was presented to our emergency department with sudden onset of seizure attacks for the first time. After controlling the seizure and stabilizing the patient, neurological examination revealed left arm paresis with motor power of $2 / 5$.
Brain CT scan and MRI revealed right frontal hemorrhage (Figure 4A). Six-vessel cerebral angiography revealed right small cortical AVM fed from distal branches of the upper division of M5 segment of the middle cerebral artery and draining to superficial cortical vein emptying into the superficial sylvian vein (Spetzler-Martin Grade I) (Figure 4B and C).

Similar to Case 1, under general anesthesia and through endovascular technique by right femoral artery access, AVM suppliers from the M5 segment were occluded with LESA. Post-embolization, angiography of the brain showed resolution of the AVM nidus (Figure 4D and E). After 4 days, the patient was operated neuronavigation-guided right frontoparietal craniotomy. During the early stage of the surgery, the feeder artery was identified with its dark color marked by LESA and cut. Total resection of the AVM in piecemeal fashion with no vascular clips and with estimated total blood loss of $150 \mathrm{~mL}$ (Figure 5A-D). Postoperatively, the boy achieved uneventful recovery without postoperative complications. The left arm power improved to normal. In addition, cerebral angiography was performed after 1 year and demonstrated total resection of the AVM (Figure 5E and F).

\section{Case 3}

A 26-year-old lady patient presented to our emergency department complaining of exacerbated headache and
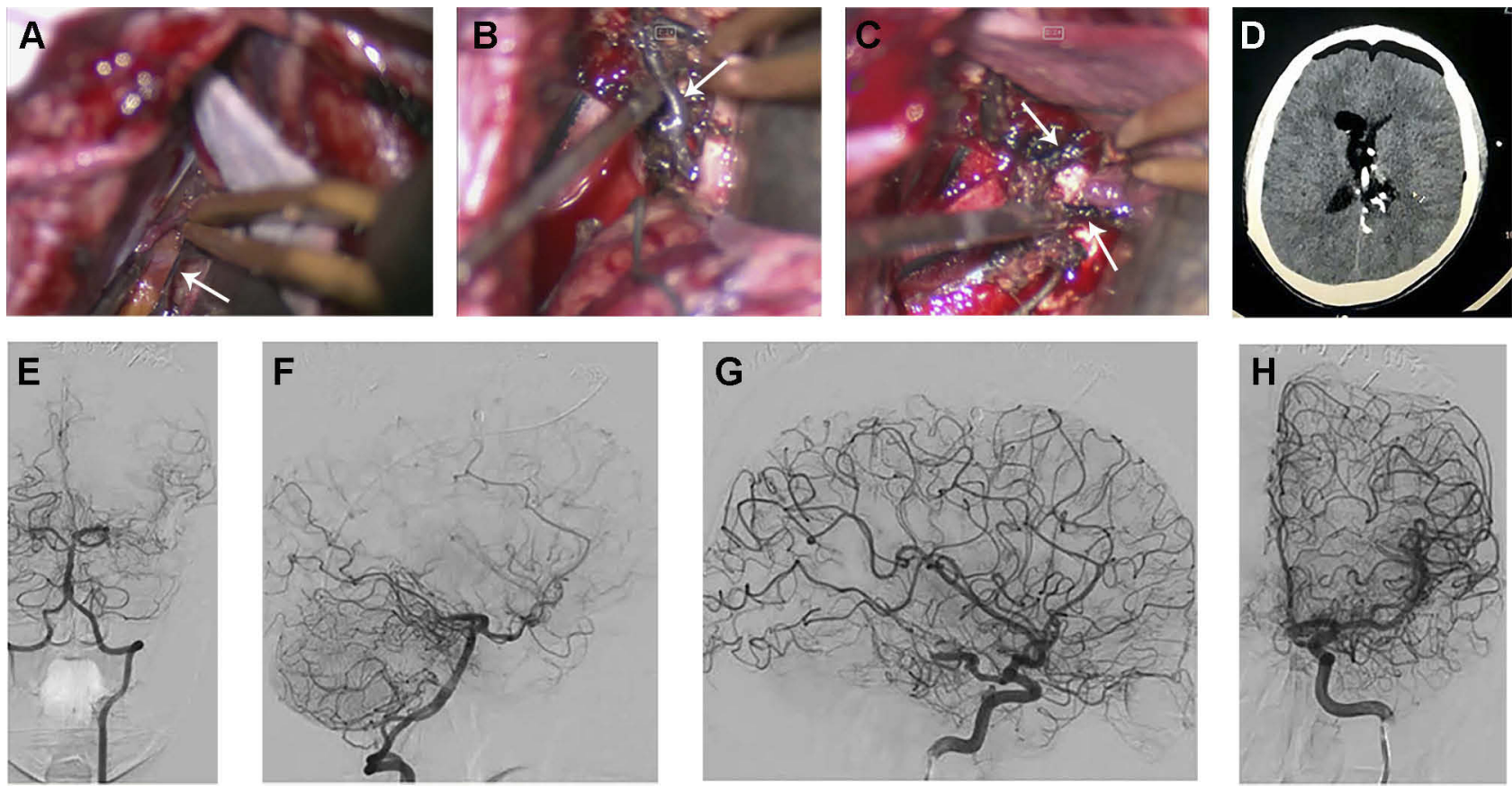

Figure 3 (A-C) Intra-operative views during surgery showing the dark embolized feeding arteries marked by Onyx ${ }^{\circledR}$ which were safely detached (arrows). Note the appearance of the nidus as a non-bleeding tuft of soft tissue. (D) Post-operative brain CT scan showing the bed of the resected AVM. Note the embolized arteries at the site of the devascularization and surgical detachment. (E-H) Post-operative cerebral angiography showing total resection of the AVM. 

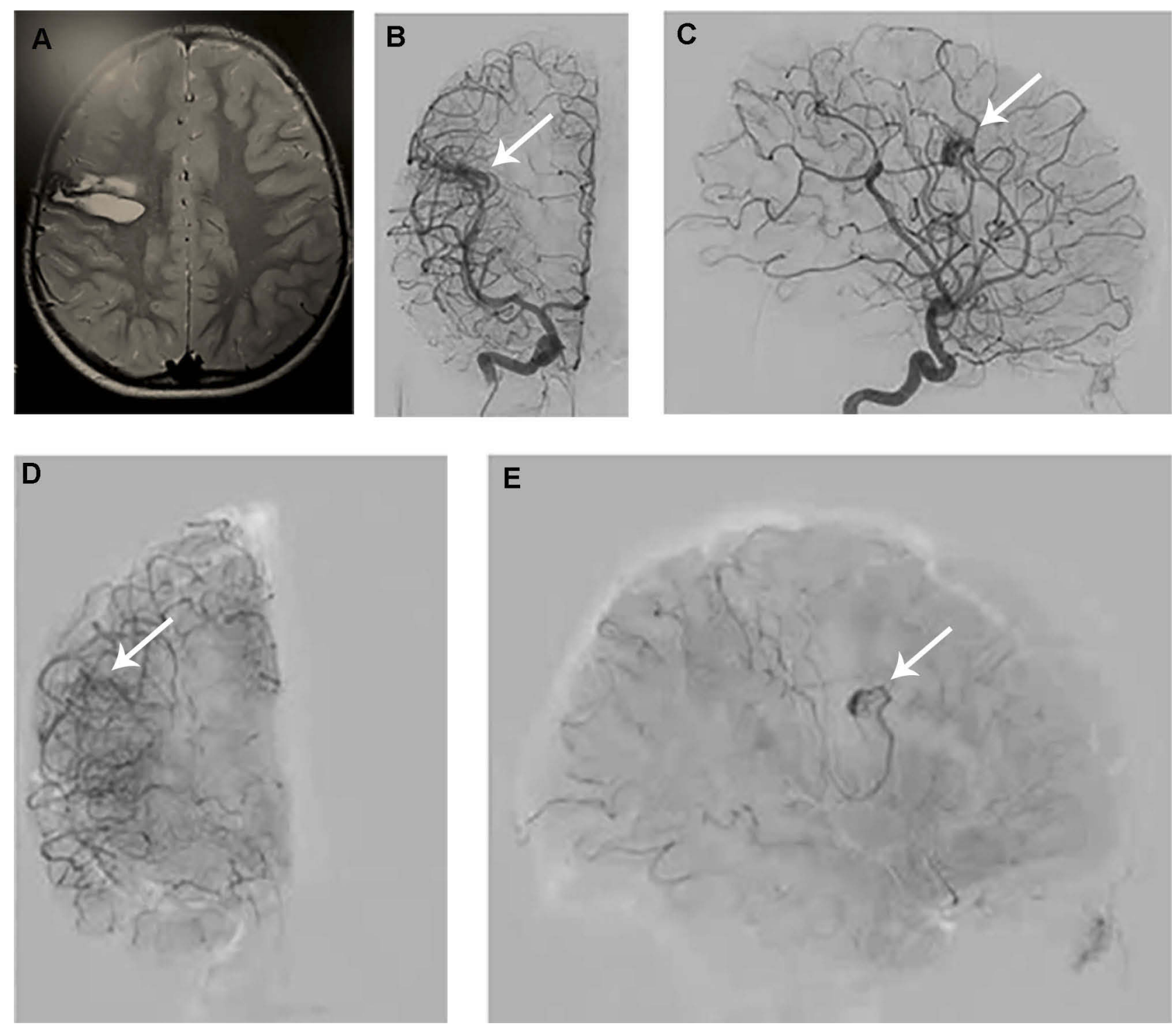

Figure 4 (A) Brain MRI showing hemorrhage in the right frontal lobe. (B and C) Cerebral angiography showing the right frontal AVM supplied by M5 branch of the right MCA and draining into a cortical vein emptying into right superficial sylvian vein. (D and $\mathbf{E}$ ) Cerebral angiography after endovascular devascularization showing the significant regress of the AVM.

swelling of the left upper eye lid. On examination, a pulsatile soft swelling near the eye led and the left frontal scalp was found without neurological deficits. MRI and CT scan revealed left frontal and periorbital soft swelling with soft tissue signal and subcutaneous flow void signs. Accordingly, six-vessel cerebral angiography was conducted and demonstrated left frontal scalp AVM fed from the external and internal carotid arteries. From the external carotid artery, it supplied from right and left superficial temporal. On the other hand, from the internal carotid artery, it is fed from the left supraorbital artery, a branch of the ophthalmic artery. The venous drainage reaches the superior sagittal sinus through a pore from the bony skull (Figure 6A-D).
Under sedative anesthesia and analgesia, through the endovascular procedure (starting from the right femoral artery), bilateral superficial temporal arteries were occluded with LESA. Post-embolization, angiography was conducted and revealed complete resolution of the nidus with remaining of the supraorbital artery as the single main feeder for the AVM (Figure 6E-G). Two days following, the dilated supraorbital artery was ligated and excised through left eyebrow incision. In addition, left fronto-temporal skin incision was created and the flap was dissected and retracted in subperiosteal fashion, leaving the AVM attached to the flap. During the retraction, the main venous drainage and bone pore was coagulated and occluded with bone wax. Dissection 

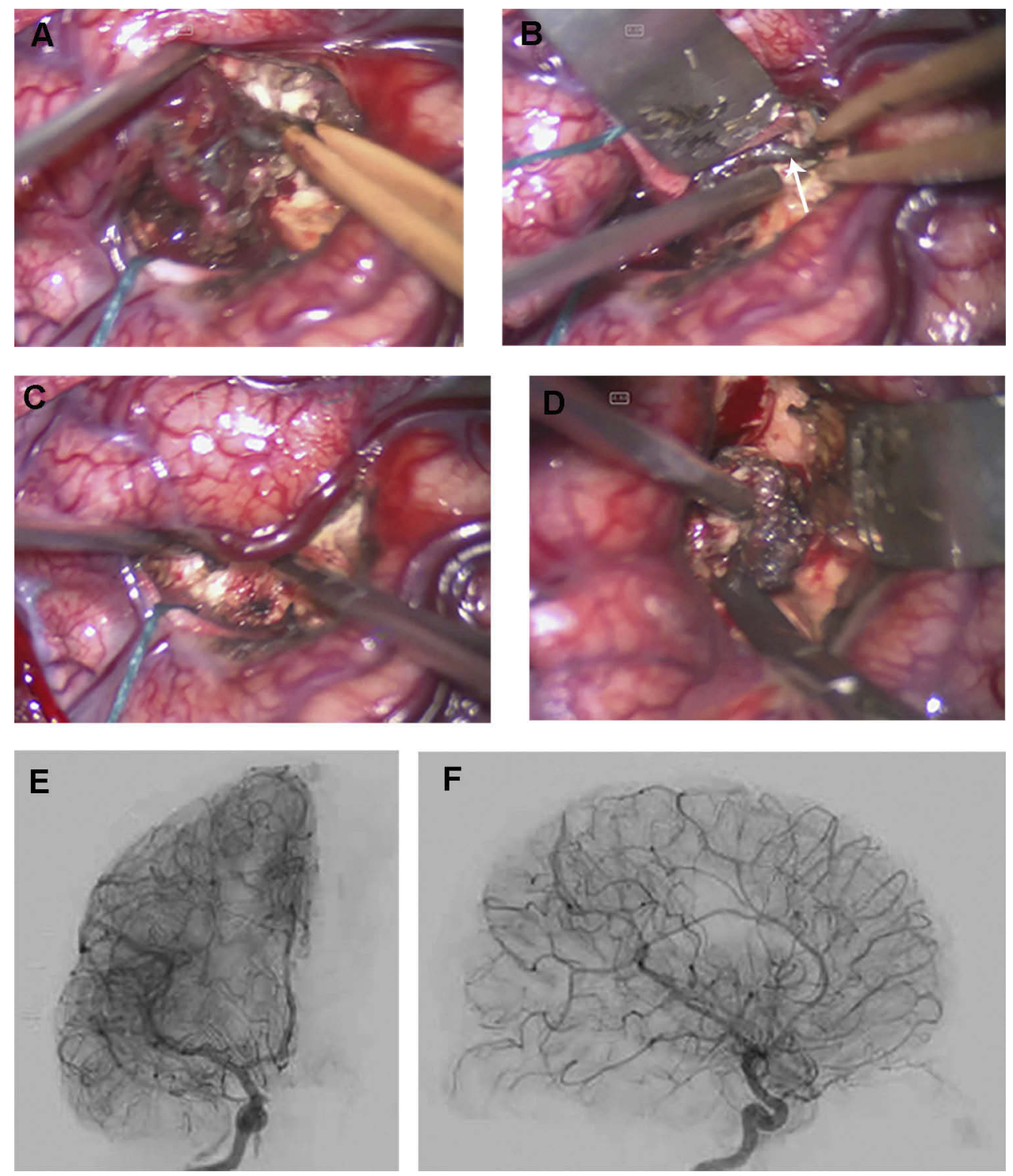

Figure 5 (A-D) Intra-operative views during surgery showing the dark embolized feeding artery which was detached (arrow). Note that the nidus was resected in piecemeal fashion. (E and F) Post-operative cerebral angiography showing total resection of the AVM.

and retraction of the flap was continued until the superior orbital rim. The AVM was sealed and dissected from the perigaleal plane to be totally resected (Figure 6A-D). The wound was closed and a drain was left beneath the flap. No intraoperative vascular clips were utilized, nor blood transfusion was needed, with blood loss of about $200 \mathrm{~mL}$ (Figure 7A-F).

Postoperatively, the patient was discharged without complications. After one-year, cerebral angiography revealed no recurrence of the AVM nidus with normal intact superior sagittal sinus flow (Figure 6E and F).

\section{Results}

Total resection of the AVM was done in all cases, without any neurologic or systemic complications. No major bleeding occurred, nor surgical clips were used during surgery. Follow-up cerebral angiography for the patients after 1 year showed complete absence of the malformations. Brain AVMs were safely resected in piecemeal fashion.

\section{Discussion}

AVM treatment is challenging and the approach for management of such cases should be multidisciplinary: a cure may be achieved by surgery, radiosurgery, neurointerventional surgery, or a combination of these methods. The endovascular approach for AVM can achieve a reduction of AVM nidus dimension prior to surgery or it can eliminate associated vascular lesion such as aneurysms and

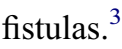



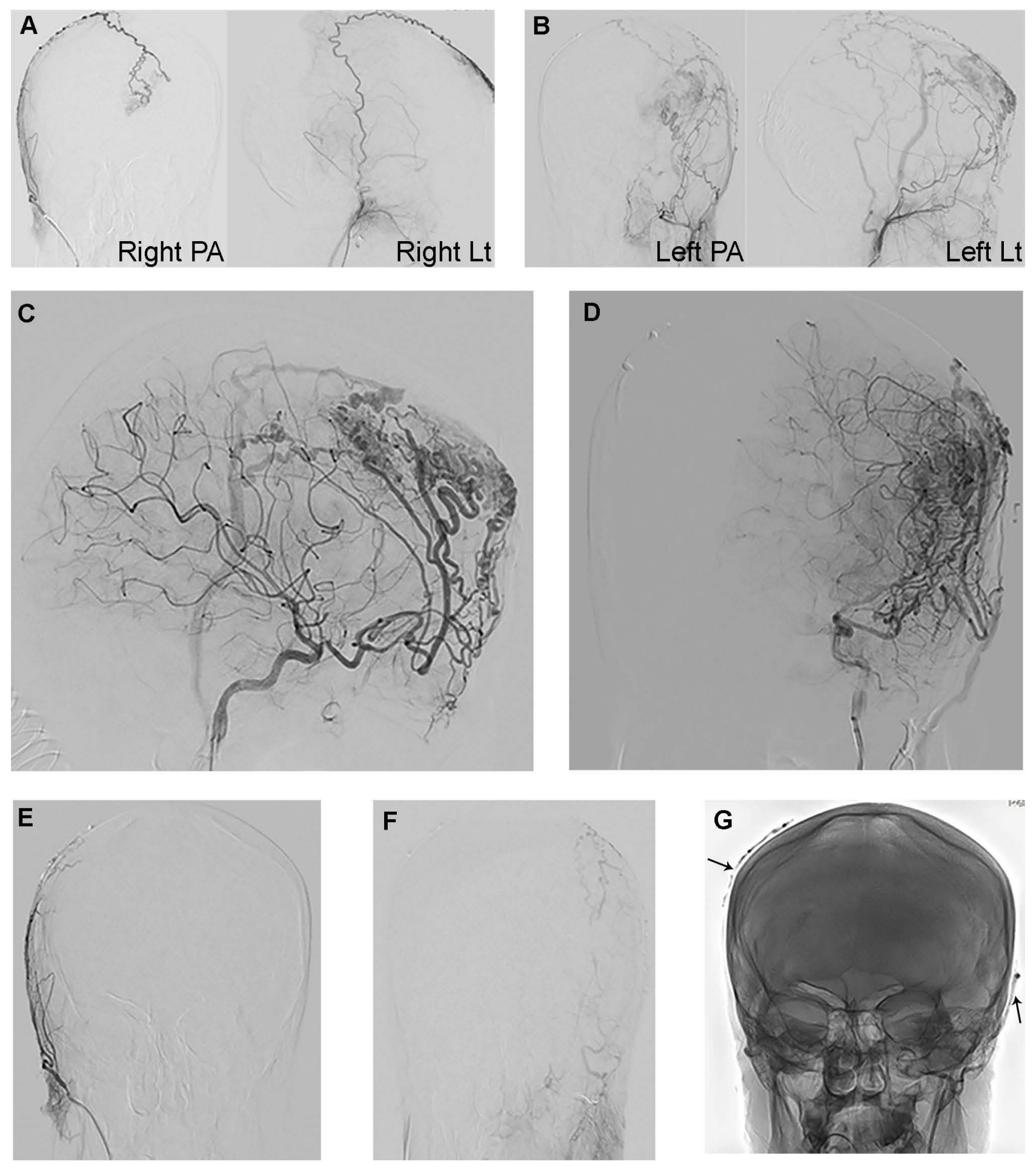

Figure 6 (A and B) Cerebral angiography of the left and right ECA's showing the arterial supply of the scalp AVM. (C and D) Cerebral angiography of the left ICA showing the AVM supply from the supraorbital artery branch of the left ophthalmic artery. (E-G) Cerebral angiography of the bilateral ECA after endovascular devascularization of bilateral superficial temporal arteries showing the significant regress of the AVM. Note the site of the devascularization (arrows).

The standard management for AVM is the surgical resection whenever possible. ${ }^{3}$ According to a study by Fleetwood and Steinberg, when surgery is performed by expert vascular neurosurgeons, $94-100 \%$ of the small nidus AVMs can achieve an angiographic cure with low morbidity rates (from $1 \%$ to $10 \%$ ). ${ }^{4}$ These percentages significantly vary with the treatment of bigger lesions located in critical brain regions, namely Spetzler and 

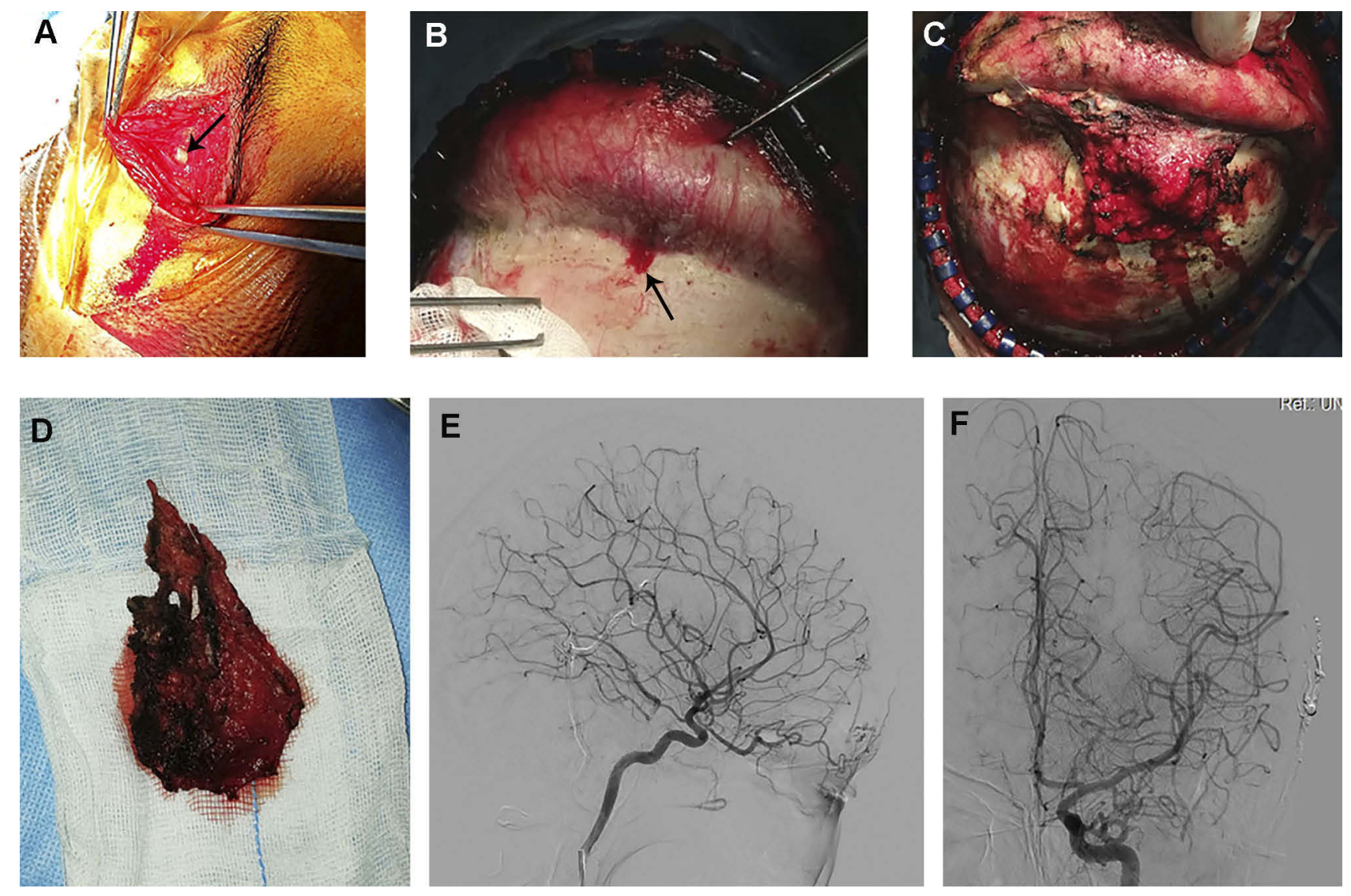

Figure 7 (A) Intraoperative picture showing the site of the left eye-brow incision and the cut of the left supraorbital artery (arrow). (B) Intraoperative picture showing the dissection of the AVM in subperiosteal plane and exposure of the draining bony venous pore (arrow). (C) Intraoperative picture showing the peri-galeal plane of the AVM dissection with noticed very minimal blood oozing from the soft tissues. (D) The AVM after resection. (E and F) Post-operative cerebral angiography of the left ICA showing the total resection of the nidus of the AVM.

Martin grade IV and V AVMs, and angiographic cure may be obtained only in $22 \%$ and $17 \%$, respectively, of the cases. ${ }^{4}$ Hartmann et al reported in their study that $3 \%$ of patients sustained surgery-related disabling neurologic symptoms and $32 \%$ of patients suffered from surgeryrelated non-disabling neurological deficits. ${ }^{5}$ van Beijnum et al stated in their meta-analysis that complete AVM occlusion can be achieved in $96 \%$ of patients who underwent microsurgery with a permanent complication rate of $7.4 \%{ }^{6}$

The advantage of utilization of pre-surgical embolization is down-grade the high inoperable Spetzler-Martin grade lesions to lower-grade operable lesions. ${ }^{7}$ Through several mechanisms, embolization can improve surgical outcomes of AVMs. These include reduction of the dimensions of the active nidus, occlusion of deep feeding arteries, and elimination of the feeding pedicle or nidal aneurysms that have either bled or are at risk of bleeding. ${ }^{7}$ Furthermore, pre-surgical embolization can act as a surgical guidance as embolized vessels are easily identifiable. Mostly, neurointerventionalist can achieve a selective embolization of the deep feeders in the opposite site of the surgical operation field through the utilization of LESA rather than glue to improve surgical resectability. New studies showed the safety and efficacy of combined endovascular embolization and microsurgical removal of AVMs in the hybrid operating room. ${ }^{8}$ Killory et al described the advantage of using green indocyanine to allow visualizing residual portions of AVMs during surgery to maximize surgical benefits. ${ }^{9}$

Although the final goal of AVM therapy is the complete obliteration of its nidus, this is not always possible. An endovascular approach can be applied in various approaches, such as embolization prior to surgery/radiosurgery, to treat vascular anomalies with an AVM (aneurysms and fistulas), as a curative therapy or in a palliation setting (ie, alleviation of blood flow steal symptoms). ${ }^{10}$ Through the most distal part of the feeding artery, nidal embolization can be performed by the microcatheter to allow the embolisate to be deposited into the nidus rather than the feeding artery. ${ }^{11,12}$ This allows the reduction of 
nidal size dimensions with no risk of persistence of the AVM and the following development of additional feeders. Moreover, it facilitates the down-grading of high-grade AVMs. As the AVMSs become lower-grade, the surgical resection or radiosurgery can be easily performed. Unfortunately, this technique is difficult to perform and needs very skilled hands. The endovascular embolization of the nidus can be performed as an adjuvant treatment before surgery due to the benefits discussed earlier. Recently, a transvenous approach has been developed and it can be done only in selected cases. These selected cases include high-flow venous side aneurysm occlusion which is not possible to navigate the microcatheter through small and tortuous arteries to reach the AVM nidus or when surgery or radiosurgery cannot be performed. ${ }^{13}$

After endovascular only approach, successful AVM obliteration occurred in 13\% cases; however, complications leading to permanent neurological deficits or death occurred in $6.6 \%$ after embolization, which is higher compared with those in studies in which adjunctive embolization was the goal. ${ }^{6,14}$ Given the high complication rate related to a primary embolization approach, the risks and benefits of such a treatment strategy should be discussed among a multidisciplinary team. According to a late literature review by Mosimann and Chapot, lack of homogenous control groups, and poor compliance to PRISMA reporting guidelines in most of the interventional literature, it was unable to obtain solid data and perform a statistical meta-analysis on the safety and effectiveness of curative embolization. ${ }^{15}$ Conger et al recommended avoidance of over-embolization of AVM feeding arteries as this technique may lead to hypertrophy of deep white matter feeders that may cause more risk of postembolization bleeding when endovascular-alone treatment is decided or in technical complexity during surgical resection when post-embolization microsurgical intervention is planned. ${ }^{3}$

Endovascular management of an AVM should not be done to reduce seizure frequency because seizures attacks themselves in AVM were not a predictor of rupture. On the other hand, endovascular procedure can cause a higher rate morbidity and mortality. ${ }^{16}$ Also, growing evidence from the literature shows that AVM bleeding is not totally excluded after even total embolization of the AVM and case reports documented LESA resorption with AVM recanalization after complete AVM obliteration. ${ }^{17,18}$

Radiosurgery can be used successfully in selected cases of high-grade AVMs (mostly IV and V grade lesions) which is considered to be inoperable or prone to severe or fatal complications if treated with other types of procedures. An important limitation of radiosurgery is the latency for devascularization results which reaches usually 2 years' time from treatment (can be up to 4 years), a period during which patients are exposed to hemorrhagic risks comparable to non-operated. ${ }^{19}$ The second limitation concerns the radiosurgical target volume effect upon adjacent structures, which may lead to an iatrogenic morbidity. ${ }^{19}$ Complete obliteration is demonstrated in $50-90 \%$ of the cases and its rate is inversely related to the AVM nidus size. ${ }^{20,21}$ Also, radiosurgery may be limited by complications leading to permanent neurological deficits or death in $5.1 \%$ of the cases. $^{6}$

In our technique, pre-surgical AVM feeder embolization is an effective and more simple technique than nidus occlusion. This is obvious in cases of diffuse-type nidus in which the total embolization is challenging. Also, this obvious in cases of direct high flow venous output into a major and/or deep vein where there is more risk of glue emboli of these veins. The post-embolization angiography and intraoperative findings showed significant reduction of the blood flow and volume in the AVM which facilitated the surgical resection without any major bleeding and nor using clips. This also facilitated the surgical resection by giving the chance of piecemeal fashion resection. The angiographic views of the AVM's in our cases are rarely seen in the general practice as the general concept is to enter and embolize the nidus -totally or partially - whenever it is possible to reach the nidus by the microcatheter. Another important advantage of this technique during surgery is previous marking (coloring) of the feeding arteries. With the previous knowledge of the surgeon about the embolized arteries and their anatomic relation to the nidus, intro-operative identification of these arteries is facilitated as these arteries look (colored) darker due to the LESA. Thus, efforts to identify the feeders by extended dissection to track these arteries, occlusion test and/ or using intraoperative ICG are reduced. Even in cases where the feeders are located in deep planes, partial resection in piecemeal fashion is applicable safely until exposing these arteries, as shown in our cases. Also, it is considered costeffective due to using less embolic agents compared to endovascular approaches aiming for partial or complete embolization of the nidus before surgery.

\section{Conclusion}

Pre-surgical endovascular proximal feeder artery devascularization technique shows to be simple, safe, and effective 
technique for the treatment of cranial arteriovenous malformations. This technique simplifies both the endovascular and surgical approaches to complicated cranial AVM cases. Also, it is considered cost-effective due to using less embolic agents compared to endovascular approaches aiming to embolize the nidus. We believe that this technique can gain popularity and can be applied to more cases that would be published in the future in the literature.

\section{Data Sharing Statement}

The datasets generated and analyzed during the current study are available from the corresponding author.

\section{Funding}

This research did not receive any specific grant from funding agencies in the public, commercial, or not-forprofit sectors.

\section{Ethics and Patient Consent}

Written informed consent was obtained from the patients for publication. Institutional approval was obtained from the Institutional Review Board at Jordan University of Science and Technology. This study was conducted in accordance with the Declaration of Helsinki.

\section{Funding}

This research did not receive any specific grant from funding agencies in the public, commercial, or not-forprofit sectors.

\section{Disclosure}

The authors report no conflicts of interest in this work.

\section{References}

1. Ajiboye N, Chalouhi N, Starke MR, et al. Cerebral arteriovenous malformations: evaluation and management. Sci World J. 2014;2014:649036.

2. Abecassis IJ, Xu DS, Batjer HH, Bendok BR. Natural history of brain arteriovenous malformations: a systematic review. Neurosurg Focus. 2014:37:E7. doi:10.3171/2014.6.FOCUS14250

3. Conger A, Kulwin C, Lawton MT, et al. Diagnosis and evaluation of intracranial arteriovenous malformations. Surg Neurol Int. 2015;6:76. doi: $10.4103 / 2152-7806.156866$

4. Fleetwood IG, Steinberg GK. Arteriovenous malformations. Lancet. 2002;359(9309):863-873. doi:10.1016/S0140-6736(02)07946-1

5. Hartmann A, Mast H, Mohr JP, et al. Determinants of staged endovascular and surgical treatment outcome of brain arteriovenous malformations. Stroke. 2005;36(11):2431-2435. doi:10.1161/01. STR.0000185723.98111.75
6. van Beijnum J, van der Worp HB, Buis DR, et al. Treatment of brain arteriovenous malformations: a systematic review and meta-analysis. JAMA. 2011;306(18):2011-2019. doi:10.1001/ jama.2011.1632

7. Bruno CA Jr, Meyers PM. Endovascular management of arteriovenous malformations of the brain. Intervent Neurol. 2012;1:109-123. doi: $10.1159 / 000346927$

8. Grüter BE, Mendelowitsch I, Diepers M, et al. Combined endovascular and microsurgical treatment of arteriovenous malformations in the hybrid operating room. World Neurosurg. 2018;117:e204-e214. doi:10.1016/j.wneu.2018.05.241

9. Killory D, Nakaji P, Gonzales LF, et al. Prospective evaluation of surgical microscope-integrated intraoperative near-infrared indocyanine green angiography during cerebral arteriovenous malformation surgery. Neurosurgery. 2009;65(3):456-462. doi:10.1227/01.NEU. $0000346649.48114 .3 \mathrm{~A}$

10. Fiorella D, Albuquerque FC, Woo HH, et al. The role of neuroendovascular therapy for the treatment of brain arteriovenous malformations. Neurosurgery. 2006;59(5Suppl 3):S163-S177. doi:10.1227/01. NEU.0000237544.20452.47

11. Krings T, Hans FJ, Geibprasert S, et al. Partial "targeted" embolisation of brain arteriovenous malformations. Eur Radiol. 2010;20:2723-2731. doi:10.1007/s00330-010-1834-3

12. van Rooij WJ, Jacobs S, Sluzewski M, et al. Curative embolization of brain arteriovenous malformations with onyx: patient selection, embolization technique, and results. Am $J$ Neuroradiol. 2012;33:1299-1304. doi:10.3174/ajnr.A2947

13. Kessler I, Riva R, Ruggiero M, et al. Successful transvenous embolization of brain arteriovenous malformations using onyx in five consecutive patients. Neurosurgery. 2011;69:184-193. doi:10.1227/ NEU.0b013e318212bb34

14. Wu EM, El Ahmadieh TY, McDougall CM, et al. Embolization of brain arteriovenous malformations with intent to cure: a systematic review. J Neurosurg. 2019;1:1-12.

15. Mosimann PJ, Chapot R. Contemporary endovascular techniques for the curative treatment of cerebral arteriovenous malformations and review of neurointerventional outcomes. J Neurosurg Sci. 2018;62(4):505-513. doi:10.23736/S0390-5616.18.04421-1

16. Lv X, Li Y, Jiang C, et al. Brain arteriovenous malformation and endovascular treatment: effect on seizures. Interv Neuroradiol. 2010;16(1):39-45. doi:10.1177/159101991001600105

17. Bauer AM, Bain MD, Rasmussen PA. Onyx resorbtion with AVM recanalization after complete AVM obliteration. Interv Neuroradiol. 2015;21(3):351-356. doi:10.1177/1591019915581985

18. Sy ECN, Melot A, Troude L, et al. Delayed hemorrhagic complication after complete embolization of a brain arteriovenous malformation. Neurochirurgie. 2018;64(4):316-320. doi:10.1016/j. neuchi.2018.04.005

19. Rubin A, Brunswick A, Riina $\mathrm{H}$, et al. Advances in radiosurgery for arteriovenous malformations of the brain. Neurosurgery. 2014;74(suppl 1):S50-S59. doi:10.1227/NEU.0000000000000 219

20. Kano H, Lunsford LD, Flickinger JC, et al. Stereotactic radiosurgery for arteriovenous malformations. Part 1. management of Spetzler-Martin grade I and II arteriovenous malformations. $J$ Neurosurg. 2012;116:11-20. doi:10.3171/2011.9.JNS101740

21. Peschillo S, Caporlingua A, Colonnese C, et al. Brain AVMs: an endovascular, surgical, and radiosurgical update. Sci World J. 2014;834931:1-6. doi:10.1155/2014/834931 


\section{Publish your work in this journal}

Vascular Health and Risk Management is an international, peerreviewed journal of therapeutics and risk management, focusing on concise rapid reporting of clinical studies on the processes involved in the maintenance of vascular health; the monitoring, prevention and treatment of vascular disease and its sequelae; and the involvement of metabolic disorders, particularly diabetes. This journal is indexed on PubMed Central and MedLine. The manuscript management system is completely online and includes a very quick and fair peerreview system, which is all easy to use. Visit http://www.dovepress. com/testimonials.php to read real quotes from published authors. 